\title{
Incremental patient care program decreases anxiety, reduces depression and improves the quality of life in patients with colorectal cancer receiving adjuvant chemotherapy
}

\author{
JIAYING $\mathrm{LI}^{1}$ and XUESONG LIU ${ }^{2}$ \\ ${ }^{1}$ Department of Colorectal Cancer Surgery, The Second Affiliated Hospital of Harbin Medical University; \\ ${ }^{2}$ Department of Nursing, The Second Affiliated Hospital of Harbin Medical University, \\ Harbin, Heilongjiang 150086, P.R. China
}

Received August 14, 2018; Accepted June 4, 2019

DOI: $10.3892 /$ etm.2019.7877

\begin{abstract}
Colorectal cancer (CRC) is a serious disease that may lead to the occurrence of anxiety and depression; however, at present, little is known about the role of care intervention in the mental health of patients with $\mathrm{CRC}$ receiving adjuvant chemotherapy. Hence, an incremental patient care program (IPCP) was designed and its effects on anxiety, depression and quality of life (QoL) in patients with CRC receiving adjuvant chemotherapy was assessed. A total of consecutivel 298 patients with $\mathrm{CRC}$ who had undergone surgery were recruited for the present randomized, controlled study. Patients were randomly assigned to the IPCP group or the control group at a 1:1 ratio. In the IPCP group, patients received IPCP and conventional care, whereas in the control group, patients received only conventional care. The sp ecific interventions included in IPCP were patient health education, physical exercise, telephone counseling, regular examinations and care activities. Anxiety, depression and QoL were assessed using the Hospital Anxiety and Depression Scale (HADS) and the European Organization for Research and Treatment of Cancer QoL Questionnaire (QLQ-C30) scale. IPCP slightly decreased the anxiety grade at month (M) 6 compared with that of the controls $(\mathrm{P}=0.070)$. The IPCP group exhibited a significant improvement in the HADS depression score at M6 vs. M0 $(\mathrm{P}<0.001)$, and the depression grade was reduced in the IPCP group compared with that in the controls $(\mathrm{P}=0.037)$. Regarding QoL, the QLQ-C30 global health status score at M6 vs. M0 was increased $(\mathrm{P}=0.035)$ and the QLQ-C30 symptoms score at M6 vs. M0 was decreased $(\mathrm{P}=0.002)$ in the IPCP group compared with that in the controls, but no difference was observed in the QLQ-C30 function score between
\end{abstract}

Correspondence to: Dr Xuesong Liu, Department of Nursing, The Second Affiliated Hospital of Harbin Medical University, 246 Xuefu Road, Harbin, Heilongjiang 150086, P.R. China

E-mail: liuxuesonge@163.com

Key words: incremental patient care program, anxiety, depression, quality of life, colorectal cancer the two groups. Subgroup analysis by tumor-nodes-metastasis stage (II or III) demonstrated similar trends to those mentioned above. In conclusion, participation in the IPCP led to a slight decrease in anxiety, and contributed to a significant reduction in depression and an improvement in QoL in patients with CRC receiving adjuvant chemotherapy.

\section{Introduction}

Colorectal cancer (CRC) has been reported as the third most common cancer type and the fourth most common cause of cancer-associated death worldwide, affecting $\sim 1.2$ individuals and leading to 600,000 deaths per year $(1,2)$. The mortality of patients with CRC has decreased in a number of countries over the last decade, which is thought to be attributed to increased CRC screening and decreased prevalence of risk factors, as well as the development and implementation of novel treatments (3). However, $50 \%$ of patients still develop metastasis and eventually succumb to their disease, and most of them develop psychological conditions at varying degrees, including anxiety and depression, and present with poor quality of life (QoL) due to the psychological burden associated with cancer diagnosis, the pain and the side effects of therapy $(4,5)$. For patients with $\mathrm{CRC}$, chemotherapy is able to decrease the recurrence risk and prolong survival to a certain extent, but most patients experience complications including pain and diarrhea; furthermore, CRC may be associated with a significant economic burden. Hence, the emotional state and social life of the patients (and their family) is frequently negatively affected, thereby leading to the occurrence of anxiety and depression (6,7). Previous studies have estimated the occurrence of post-surgical anxiety and depression in patients with CRC to be 8-23 and 16-39\%, respectively $(5,8-10)$. Therefore, it is necessary to explore strategies to decrease anxiety/depression and improve or maintain good QoL in patients with CRC.

Care intervention has been considered a common and efficacious method to ameliorate the adverse effects of carcinoma and its treatment, and is usually designed to promote improvements in physical and mental health (11). A large number of studies have confirmed the favorable effect of care intervention, including patients' health education, dietary patterns and 
physical activity on the functional outcomes in patients with CRC; however, most of them focus on the functional outcomes in these patients, while the effect of care intervention on the prognosis of patients with CRC remains to be determined, particularly regarding psychosocial problems and QoL $(12,13)$. Although parts of Chinese clinical trials have revealed the efficacy of intervention care in improving mental health issues including anxiety and depression in CRC patients, the role of the care intervention in patients with $\mathrm{CRC}$ receiving adjuvant chemotherapy remains elusive $(14,15)$. In addition, most of these previous studies have been performed on relatively small cohorts, and additional studies with a larger sample size for validation are urgently required. Considering the aforementioned points, an incremental patient care program (IPCP) was designed, consisting of patient health education, physical exercise, telephone counselling, regular examination as well as care activities, and the purpose of the present study was to assess the effects of IPCP on anxiety, depression and QoL in patients with $\mathrm{CRC}$ receiving adjuvant chemotherapy.

\section{Materials and methods}

Participants. A total of 298 consecutive patients with CRC who underwent surgery at the 2nd Affiliated Hospital of Harbin Medical University (Harbin, China) between January 2014 and December 2016 were recruited for the present randomized, controlled study. The inclusion criteria were as follows: a) Diagnosis of primary CRC confirmed by clinicopathologic examinations; b) age of 18-80 years; c) tumor-nodes-metastasis (TNM) stage, II or III; d) scheduled for CRC resection followed by adjuvant chemotherapy; e) prognosis of survival for at least 12 months; and f) ability to complete the anxiety, depression and QoL assessments. The exclusion criteria were as follows: a) Neoadjuvant therapies; b) intake of anti-depressant or anti-anxiety medication within the past 3 months; c) a comorbidity of severe primary mental disorder, or severe liver or renal disease; d) history of other malignant tumor types or hematological malignancy; e) no availability for regular follow-up; f) pregnancy or lactation; g) refusal to provide written informed consent. The reasons for the collection of data over a long period (from January 2014 to December 2016) were as follows: First, at our hospital, numerous patients were diagnosed at the early stage and did not require adjuvant chemotherapy, and certain patients at the advanced stage received neoadjuvant therapy; thus, a considerable proportion of patients were not eligible for the present study. Furthermore, 300 patients were required to reach sufficient statistical power, and it took this long until the number of eligible consecutive patients collected reached this number. In addition, at the preliminary stage, a total of 625 patients were invited to participate in the present study, but a considerable amount were excluded due to not satisfying the inclusion or exclusion criteria. There were 5 patients with severe depressive disorder and 11 patients with severe anxiety disorder who were excluded, and these patients received psychotherapy, but no anti-depressant or anti-anxiety medicines.

Randomization. In the present randomized, controlled study, a blocked randomization method was adopted, and a randomization code was generated using Statistical Analysis System
(SAS) 9.3 software (SAS Institute, Carey, NC, USA). The randomization was performed by an independent statistical analyst who was otherwise not involved in the study, and the documents were deposited at the medical and statistical service company Shanghai Qeejen Biotech Co. (Shanghai, China). When a patient was eligible for the study, a call was made to Qeejen Biotech Co. and a unique subject identification number was provided from the randomizing module.

Adjuvant chemotherapy. At 2-6 weeks following CRC resection, all patients received adjuvant chemotherapy based on their disease condition. For patients with TNM stage II, according to risk stratification and T stage, one of the following adjuvant chemotherapy regimens was selected: i) Capecitabine $1,250 \mathrm{mg} / \mathrm{m}^{2}$ twice daily on days $1-14$, repeated every 3 weeks for a total of 24 weeks; ii) leucovorin (LV) $400 \mathrm{mg} / \mathrm{m}^{2}$ over $2 \mathrm{~h}$ on day 1 , followed by a 5-fluorouracil (5-FU) bolus $400 \mathrm{mg} / \mathrm{m}^{2}$ and then $1,200 \mathrm{mg} / \mathrm{m}^{2} /$ day for 2 days (a total continuous infusion of 2,400 mg/m² over 46-48 h), repeated every 2 weeks for a total of 24 weeks; iii) oxaliplatin $130 \mathrm{mg} / \mathrm{m}^{2}$ intravenously (IV) over $2 \mathrm{~h}$ on day 1 and capecitabine $1,000 \mathrm{mg} / \mathrm{m}^{2}$ twice daily on days $1-14$, repeated every 3 weeks for a total of 24 weeks; iv) oxaliplatin $85 \mathrm{mg} / \mathrm{m}^{2} \mathrm{IV}$ over $2 \mathrm{~h}$ on day 1 , LV $400 \mathrm{mg} / \mathrm{m}^{2} \mathrm{IV}$ over $2 \mathrm{~h}$ on day 1,5 -FU $400 \mathrm{mg} / \mathrm{m}^{2} \mathrm{IV}$ bolus on day 1 and then $1,200 \mathrm{mg} / \mathrm{m}^{2} /$ day for 2 days (total $2,400 \mathrm{mg} / \mathrm{m}^{2}$ over $46-48 \mathrm{~h}$ ) continuous infusion, repeated every 2 weeks for a total of 24 weeks. For patients with TNM stage III, one of following adjuvant chemotherapy regimens was selected: i) Oxaliplatin $130 \mathrm{mg} / \mathrm{m}^{2} \mathrm{IV}$ over $2 \mathrm{~h}$, day 1 , capecitabine $1,000 \mathrm{mg} / \mathrm{m}^{2}$ twice daily on days $1-14$, repeated every 3 weeks for a total of 24 weeks; ii) oxaliplatin $85 \mathrm{mg} / \mathrm{m}^{2} \mathrm{IV}$ over $2 \mathrm{~h}$ on day $1, \mathrm{LV}$ $400 \mathrm{mg} / \mathrm{m}^{2}$ IV over $2 \mathrm{~h}$ on day $1,5-\mathrm{FU} 400 \mathrm{mg} / \mathrm{m}^{2} \mathrm{IV}$ bolus on day 1 , then $1,200 \mathrm{mg} / \mathrm{m}^{2} /$ day for 2 days (a total continuous infusion of 2,400 mg/m $\mathrm{m}^{2}$ over $46-48 \mathrm{~h}$ ) repeated every 2 weeks for a total of 24 weeks.

Interventions. After randomization, patients were randomly assigned to the IPCP group or the control group in a 1:1 ratio. In the IPCP group, patients received IPCP and conventional care, whereas in the control group, patients received only conventional care, which included instructions of post-operative medicine management, regular examinations (every 3 months) and usual advice regarding post-operative rehabilitation. The intervention was performed for six months. The details of IPCP were as follows (Table I): i) Patient health education: Over the first 2 weeks, patients were provided with general health education materials, including information about nutrition, physical activity and mental health care. Furthermore, comprehensive health education was provied by a designated research therapist, and detailed instruction was given once every two months for six months. ii) Physical exercise: Over the first 3 months, patients participated in low-intensity physical exercise comprised of three components: Relaxation (30 min five times a week), body awareness and restorative exercise (90 min once a week) and massage (30 min twice a week). Over the subsequent 3 months, patients participated in high-intensity physical exercise for $90 \mathrm{~min}$ followed by $30 \mathrm{~min}$ of relaxation exercise three times a week. The high-intensity physical exercise sessions comprised three components: $30 \mathrm{~min}$ of warm-up exercises, 45 min of resistance exercise and 15 min of cardiovascular exercise. All physical exercise was supervised by 
Table I. Contents of the incremental patient care program.

\begin{tabular}{lll}
\hline Item & \multicolumn{1}{c}{ Frequency and duration } & \multicolumn{1}{c}{ Core content } \\
\hline $\begin{array}{l}\text { Patient health education } \\
\text { Physical exercise }\end{array}$ & $\begin{array}{l}\text { Once every 2 months for 6 months } \\
\text { Low-intensity physical exercise for } \\
3 \text { months, followed by high-intensity } \\
\text { physical exercise for 3 months }\end{array}$ & $\begin{array}{l}\text { Training for knowledge of disease and self-care } \\
\text { Recovery of physical function }\end{array}$ \\
Telephone counselling & $\begin{array}{l}\text { Weekly for 3 weeks, every other week } \\
\text { for 1 month and monthly for 4 months } \\
\text { Regular examination }\end{array}$ & Once every 2 months for 6 months \\
Care activities & Monthly for 6 months & $\begin{array}{l}\text { Surveillance for disease } \\
\text { Close communication with patients to help them }\end{array}$ \\
& & to solve problems
\end{tabular}

trained specialist nurses. Details on low- and high-intensity physical exercise are provided in Table SI. iii) Telephone counseling: Each participant was assigned a nurse for the 6-month study period. Counseling sessions were performed weekly during the first three weeks, every other week for one month thereafter and then monthly. Each telephone session had a duration of 15-30 min and served to provide social support and enhance self-efficacy (encourage patients to actively perform activities to promote their health). During each telephone call, the nurse worked with the patient to monitor progress, explore strategies for overcoming barriers and asked relevant questions about rehabilitation. iv) Regular examination: Patients were instructed to regularly undergo the scheduled examinations, including imaging, blood analysis and colonoscopy, once every two months for six months to manage any abnormalities. v) Care activities: Patients and family members as the primary caregivers were invited to the join the monthly workshop and the nurse communicated with them to resolve issues that they encountered in the interim period and provided detailed advice on points including how to maintain a healthy diet, control body weight and keep a positive mood. In addition, during the care activities, caregivers were provided with lessons regarding how to help patients complete their daily physical exercise.

Information collection. After enrolment in the present study, baseline information was collected from all patients, which included the following: a) Demographic characteristics: Age, gender and highest education; and b) Clinical and pathological characteristics: Smoking and drinking status, hypertension, hyperlipidemia, diabetes, pathological grade, tumor size and TNM stage (according to the 7th edition of the American Joint Committee on Cancer Staging Manual) (16). In the present study, the personal information of the patients, including the demographic and clinicopathological characteristics, were collected from the patients' medical records, and the Hospital Anxiety and Depression Scale-anxiety (HADS-A; Table SII), HADS-depression (HADS-D; Table SII) as well as QoL score (Table SIII) were from questionaires completed at the clinic.

Evaluation of anxiety and depression. Anxiety refers to an emotion that is featured by an unpleasant state of inner turmoil and is often accompanied by nervous behavior, including pacing back and forth, somatic complaints and rumination, or unpleasant feelings of dread over anticipated events (17). Depression refers to a state of low mood and aversion to activity, which may affect a person's thoughts, behavior (sucha as total loss of interest in enjoyable activities or socializing), feelings (such as feelings of loneliness, sadness, guilt or worthlessness) and sense of well-being (18). HADS-A scores and HADS-D scores were determined to assess anxiety and depression among the patients. These were assessed at baseline [month 0 (M0)], M1, M3 and M6. The HADS-A and HADS-D subscales consisted of seven questions that are scored individually from 0 to 3 points, resulting in subscale scores ranging from 0 to 21 points, with the following grade classification: 0-7, no anxiety/depression; 8-10, light anxiety/depression; 11-14, moderate anxiety/depression; 15-21, severe anxiety/depression (19).

Evaluation of QoL. QoL refers to the satisfaction of patients, outlining negative and positive features of life. (20). The European Organization for Research and Treatment of Cancer Quality of Life Questionnaire (EORTC QLQ-C30 Scale) was used to assess the QoL of patients. The EORTC QLQ-C30 Scale consists of 30 items, including five functions, symptoms and financial implications subscales. The first 28 items of the questionnaire used a 4-point Likert-type response scale ranging from 1 (not at all) to 4 (very much). Items 29 and 30, which assess global health status/QoL, use a response scale ranging from 1 (very poor) to 7 (excellent). All raw data were transformed to a 0-100-point scale. Higher mean scores for the functional scales and the global health status/QoL scales indicate better functioning and overall QoL, whereas a high score for the symptom scale/single-item scale represents a high level of symptom distress (21).

Statistical analysis. Sample size calculation was based on a previous study (6). Under the requirement of a $90 \%$ statistical power to detect a difference of $10 \%$ in the percentage of patients with depression at M6 between the IPCP group and control group, assuming $25 \%$ patients in the IPCP group and $35 \%$ patients in the control group exhibited depression at M6, with a two-sided 5\% level of significance $(\alpha)$, a sample size of 110 participants was required in each group. Accounting for loss to follow-up of $\sim 26 \%, 149$ patients were enrolled in each group. Statistical analyses were performed 


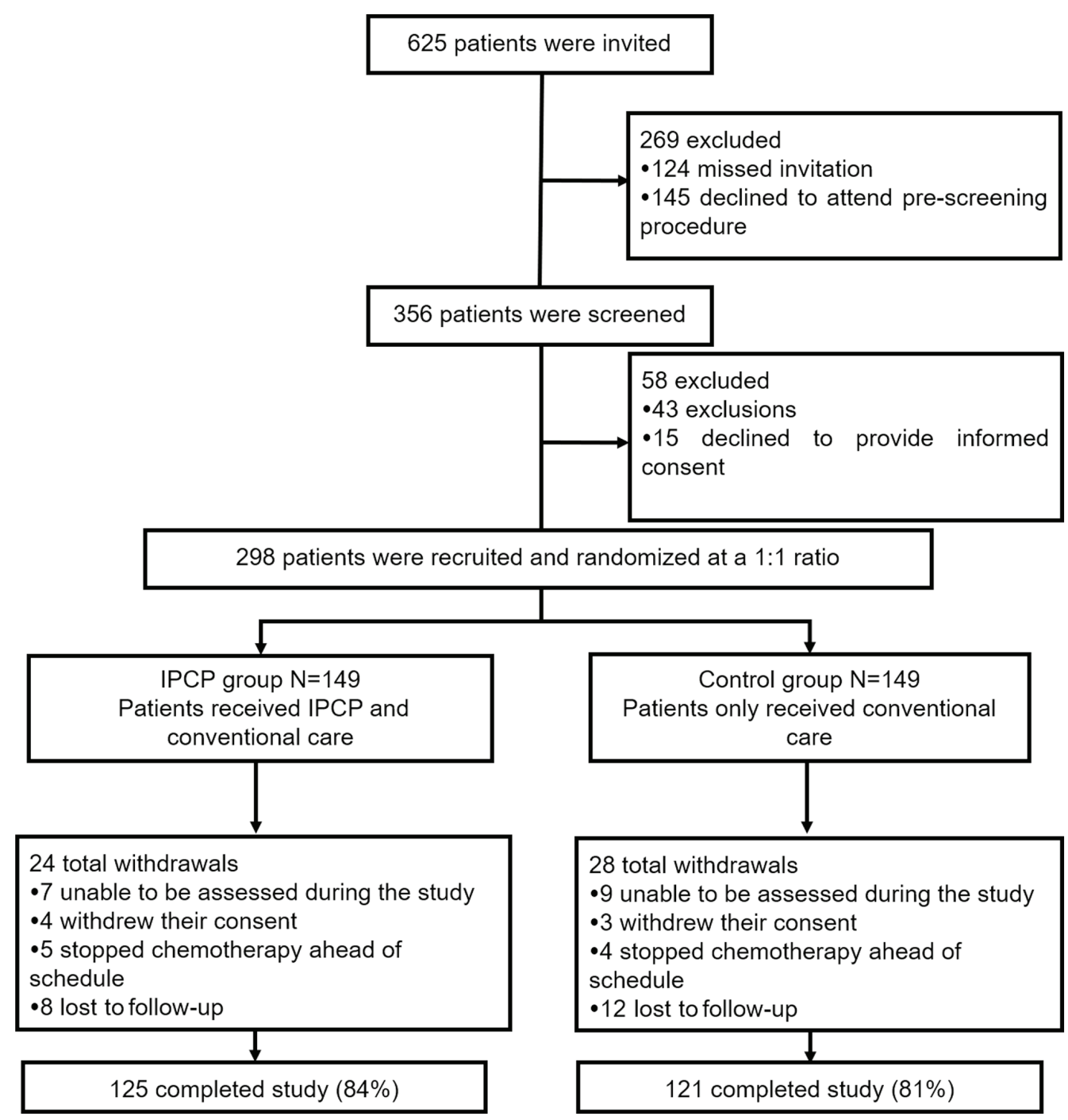

Figure 1. Flow chart depicting the movement of the subjects throughout the study. IPCP, incremental patient care program.

using SPSS 22.0 software (IBM Corp., Armonk, NY, USA), Office 2010 software (Microsoft Corp., Redmond, WA, USA) and GraphPad prim 6.0 (GraphPad Inc., La Jolla, CA, USA). Values are expressed as the mean \pm standard deviation or $\mathrm{n}(\%)$. Comparisons between groups were performed using Student's t-tests or Chi-square tests. Correlations were determined using Pearson correlation analysis. In addition, the statistics were based on an intent-to-treat analysis (22). $\mathrm{P}<0.05$ was considered to indicate statistical significance.

\section{Results}

Study flow. A total of 625 patients were invited to participate in the present study, of which while 269 patients were excluded, including 124 who missed their invitation and 145 who declined to attend the pre-screening. A total of 356 patients screened for eligibility, 58 of which were excluded, including 43 who did not meet all of the inclusion criteria and 15 who refused to provide informed consent. The remaining 298 patients were then randomized at a 1:1 ratio. Among these, 149 received IPCP and conventional care (IPCP group), whereas the other 149 received only conventional care (control group). In the IPCP group, 24 patients dropped out during the study period, including 7 who were unable to be assessed during the study, 4 who withdrew their consent, 5 who stopped chemotherapy ahead of schedule and 8 who were lost to follow-up, resulting in 125 patients (84\%) who completed the entire study. In the control group, there were a total of 28 dropouts, including 9 cases who were unable to be assessed during the study, 3 who withdrew their consent, 4 who stopped chemotherapy ahead of schedule and 12 who were lost to follow-up, resulting in 121 patients $(81 \%)$ who ultimately completed the study (Fig. 1). In the present study, the statistics were based on an intent-to-treat analysis (22). For patients who did not complete the entire study, the last available values were used to represent subsequent missing evaluation indexes.

Baseline characteristics. The baseline characteristics of the patients in the control and IPCP groups are provided in Table II. No differences were observed in demographic and clinical characteristics between the IPCP group and the control group (all $\mathrm{P}>0.05$ ). The mean age was $60.06 \pm 11.00$ years in the IPCP group and $58.47 \pm 12.52$ years in the control group. The amount 
Table II. Baseline characteristics of patients in the IPCP group and the control group.

\begin{tabular}{|c|c|c|c|}
\hline Item & IPCP group (n=149) & Control group $(n=149)$ & P-value \\
\hline Age (years) & $60.06 \pm 11.00$ & $58.47 \pm 12.52$ & 0.245 \\
\hline Gender (male/female) & $98 / 51$ & $91 / 58$ & 0.400 \\
\hline Highest education & & & 0.362 \\
\hline Primary school or less & $74(48.3)$ & $73(49.0)$ & \\
\hline High school & $42(28.2)$ & $50(33.6)$ & \\
\hline Undergraduate & $35(23.5)$ & $26(17.4)$ & \\
\hline Graduate or above & $0(0.0)$ & $0(0.0)$ & \\
\hline Smoking & $58(38.9)$ & $68(45.6)$ & 0.241 \\
\hline Drinking & $57(38.3)$ & $60(40.3)$ & 0.722 \\
\hline Hypertension & $48(32.2)$ & $58(38.9)$ & 0.226 \\
\hline Hyperlipidemia & $35(23.5)$ & $36(24.2)$ & 0.892 \\
\hline Diabetes & $11(7.4)$ & $14(9.4)$ & 0.531 \\
\hline Pathological grade & & & 0.466 \\
\hline 1 & $14(9.4)$ & $13(8.7)$ & \\
\hline 2 & $88(59.1)$ & $98(65.8)$ & \\
\hline 3 & $47(31.5)$ & $38(25.5)$ & \\
\hline Tumor size (cm) & $4.68 \pm 1.28$ & $4.67 \pm 1.34$ & 0.940 \\
\hline TNM stage & & & 0.104 \\
\hline II & $72(48.3)$ & $86(57.7)$ & \\
\hline III & $77(51.7)$ & $63(42.3)$ & \\
\hline \multicolumn{4}{|l|}{ HADS-anxiety score } \\
\hline No anxiety (score, $0-7$ ) & $110(73.8)$ & $118(79.2)$ & 0.274 \\
\hline Anxiety (score, 8-21) & $39(26.2)$ & $31(20.8)$ & \\
\hline HADS-anxiety grade & & & 0.308 \\
\hline Light grade ( 8-10 score) & $26(17.4)$ & $15(10.1)$ & \\
\hline Moderate grade (11-14 score) & $11(7.5)$ & $13(8.7)$ & \\
\hline Severe grade (15-21 score) & $2(1.3)$ & $3(2.0)$ & \\
\hline \multicolumn{4}{|l|}{ HADS-depression score } \\
\hline No depression (score, $0-7$ ) & $98(65.8)$ & $103(69.1)$ & 0.536 \\
\hline Depression (score, 8-21) & $51(34.2)$ & $46(30.9)$ & \\
\hline \multicolumn{4}{|l|}{ HADS-depression grade } \\
\hline Light (score, 8-10) & $33(22.1)$ & $24(16.1)$ & 0.278 \\
\hline Moderate (score, 11-14) & $15(10.1)$ & $14(9.4)$ & \\
\hline Severe (score, 15-21) & $3(2.0)$ & $8(5.4)$ & \\
\hline \multicolumn{4}{|l|}{ EORTC QLQ-C30 scale } \\
\hline Global Health Status score & $63.7 \pm 14.5$ & $62.9 \pm 15.8$ & 0.630 \\
\hline Functions score & $69.9 \pm 16.5$ & $69.9 \pm 18.9$ & 0.995 \\
\hline Symptoms score & $33.3 \pm 16.9$ & $30.8 \pm 14.9$ & 0.189 \\
\hline
\end{tabular}

Values are expressed as the mean value \pm standard deviation or $\mathrm{n}(\%)$. IPCP, incremental patient care program; HADS, Hospital Anxiety and Depression Scale; TNM, tumor-nodes-metastasis; EORTC QLQ-C30, European Organization for Research and Treatment of Cancer Quality of Life Questionnaire.

of patients with hypertension, hyperlipidemia and diabetes was 48 (32.2\%), 35 (23.5\%) and $11(7.4 \%)$, respectively, in the IPCP group and $58(38.9 \%), 36(24.2 \%)$ and $14(9.4 \%)$, respectively, in the control group. In addition, there were no differences between groups in HADS-A scores, HADS-A grade, HADS-D score, HADS-D grade and EORTC QLQ-C30 scale scores (all $\mathrm{P}>0.05)$.
Comparison of anxiety scores between the IPCP group and control group. There were no differences between groups in HADS-A scores at each visit (all P>0.05; Fig. 2A), HADS-A change score from baseline (M0) to M6 ( $\mathrm{P}=0.289$; Fig. 2B) or in the percentage of patients with anxiety according to HADS-A score at M6 ( $\mathrm{P}=0.381$; Fig. 2C). Compared with that in the control group, the anxiety score at M6 in IPCP 

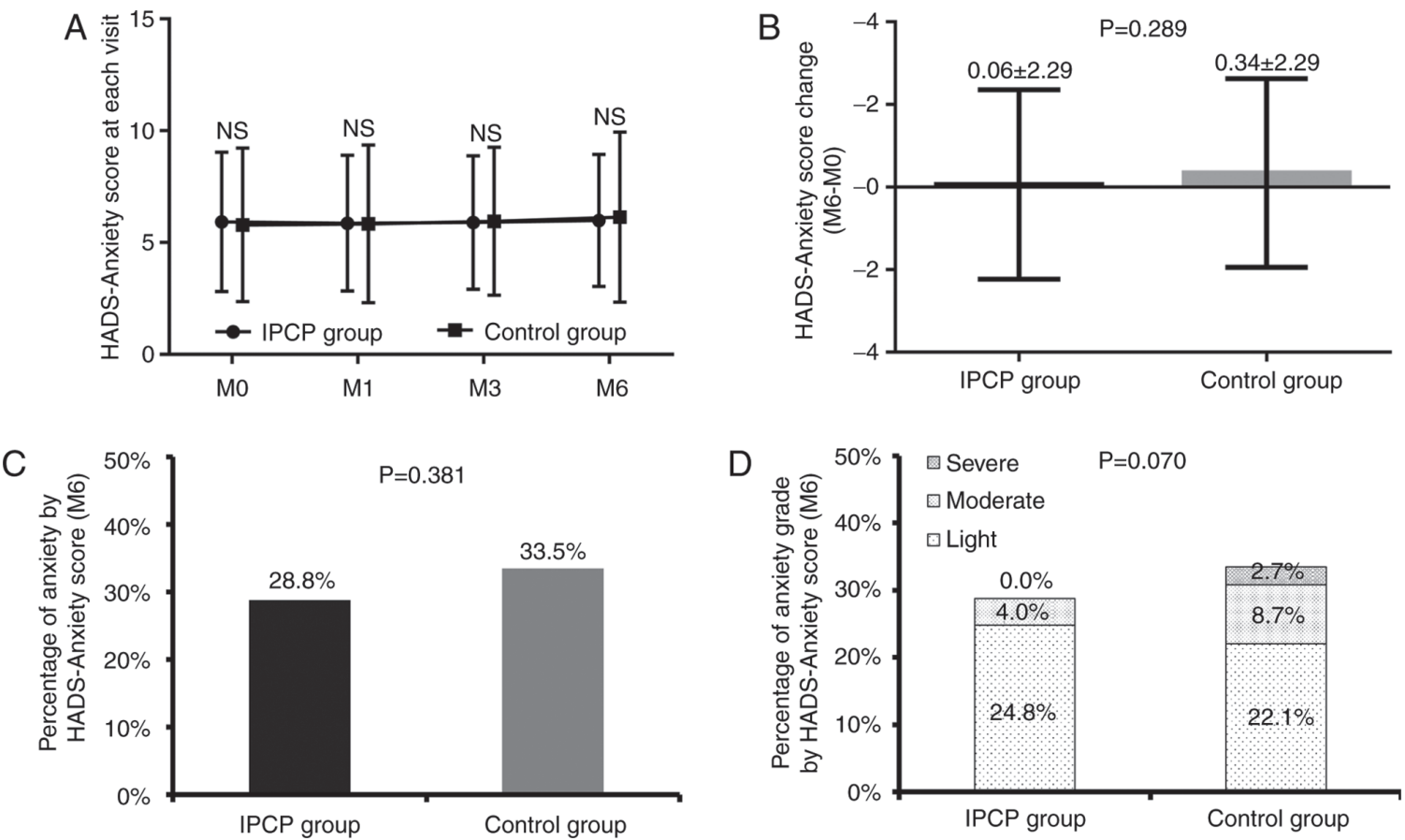

Figure 2. Comparison of anxiety scores between IPCP group and control group. (A) HADS-anxiety score at each visit; no differences were observed. (B) HADS-anxiety score change (M6-M0) and (C) the Percentage of anxiety assessed by HADS-anxiety score between groups. (D) IPCP slightly decreased the anxiety grade at month 6 compared with the control group. Comparison was performed using the t-test or Chi-square test. Values are expressed as the mean \pm standard deviation. NS, no significance; IPCP, incremental patient care program; HADS, Hospital Anxiety and Depression Scale; M, month.

participants appeared to have decreased but the difference was not statistically significant ( $\mathrm{P}=0.070$; Fig. 2D).

Comparison of depression scores between the IPCP group and control group. No differences were identified in HADS-D scores at M0, M1 or M3 between the IPCP and control groups (all P>0.05; Fig. 3A), although they were lower in the IPCP group than those in the control group at $\mathrm{M} 6(\mathrm{P}<0.05$; Fig. $3 \mathrm{~A})$. As for HADS-D change scores (M6-M0) between the two groups, they were decreased in the IPCP group compared with those in the control group $(\mathrm{P}<0.001$; Fig. $3 \mathrm{~B})$. There was no difference between the groups in the percentage of participants with depression at M6 according to their HADS-D scores $(\mathrm{P}=0.284$; Fig. 3C). In addition, the depression grade was reduced in the IPCP group compared with the control group $(\mathrm{P}=0.037$; Fig. 3D).

Comparison of QoL between the IPCP group and control group. No difference in the QLQ-C30 global health status scores was identified between the IPCP and control groups at M0, M1 or M3 (all P>0.05; Fig. 4A), although they were higher in the IPCP group at M6 compared with those in the control group ( $\mathrm{P}<0.05$; Fig. 4A). The QLQ-C30 global health status change scores (M6-M0) exhibited a greater increase in the IPCP group compared with that in the control group $(\mathrm{P}=0.035$; Fig. 4B). Regarding the QLQ-C30 function scores, there were no differences between the groups at each visit (all $\mathrm{P}>0.05$; Fig. 4C), and there was also no significant difference in the change score (M0-M6) between the two groups $(\mathrm{P}=0.105$; Fig. 4D). As for the QLQ-C30 symptom scores, no differences between the groups were present at each visit (all $\mathrm{P}>0.05$ ), but the QLQ-C30 symptom change scores (M6-M0) were decreased in the IPCP group compared with those in the control group $(\mathrm{P}=0.002$; Fig. 4F).

Subgroup analysis between TNM stage II and III patients. To assess the impact of the TNM stage on the above analysis, the patients were stratified into TNM stage II and III groups (Fig. 5). For TNM stage II patients, the HADS-D change scores (M6-M0) ( $\mathrm{P}=0.002$; Fig. 5B) and QLQ-C30 symptom change scores (M6-M0) $(\mathrm{P}=0.050$; Fig. 5E) were decreased in the IPCP group compared with those in the control group. However, no difference was observed in the change in HADS-A scores ( $\mathrm{P}=0.464$; Fig. 5A), QLQ-C30 global health status scores $(\mathrm{P}=0.264$; Fig. 5C) or QLQ-C30 function scores $(\mathrm{P}=0.544$; Fig. 5D) from baseline to M6 between the two groups.

For TNM stage III patients, the QLQ-C30 symptom scores (M6-M0) exhibited a greater decrease in the IPCP group compared with those in the control group $(\mathrm{P}=0.010$; Fig. 5J), as did the HADS-depression change scores (M6-M0) $(\mathrm{P}=0.054$; Fig. 5G), whereas the QLQ-C30 global health status scores (M6-M0) ( $\mathrm{P}=0.065$; Fig. 5H) exhibited a greater increase in the IPCP group compared with those in the control group. There was no difference in the change of HADS-A scores or QLQ-C30 function scores from baseline to M6 between the groups ( $\mathrm{P}=0.129$; Fig. $5 \mathrm{~F}$ and $\mathrm{I})$. The effect of IPCP intervention on anxiety, depression and QoL scores and their rate is also provided in Tables SIV and SV. In addition, changes in anxiety, depression and QoL score in the subgroups are also presented in Table SVI. Furthermore, correlations between HADS-A score, HADS-D score and QLQ-C30 scores at M0 and M6 are displayed in Table SVII; however, no significant differences were identified in control group at baseline and at 6 months. 
A

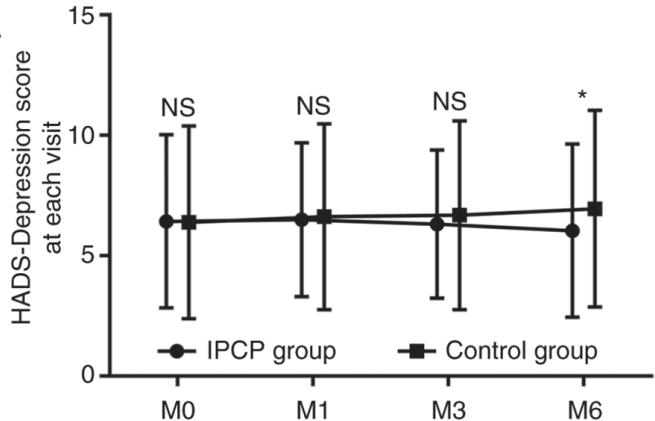

C

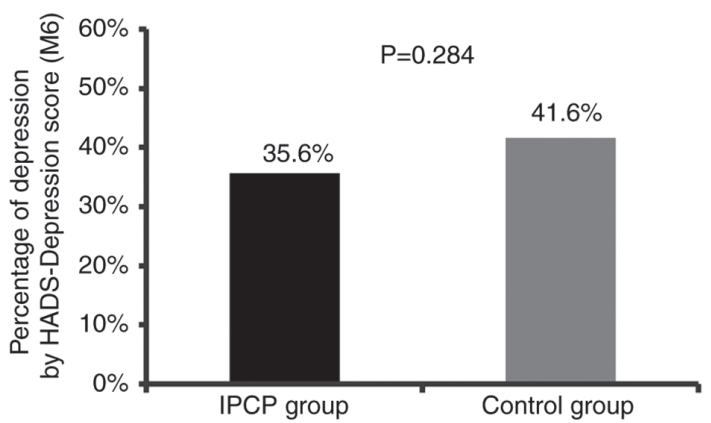

B
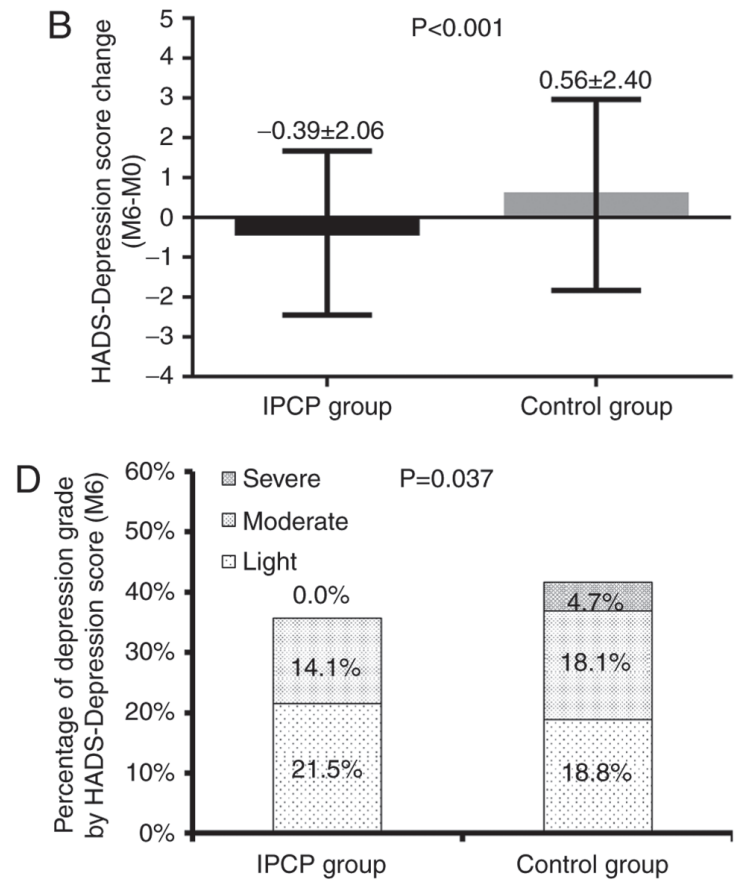

Figure 3. Comparison of depression scores between IPCP group and control group. (A) The HADS-depression score was lower in the IPCP group compared with that in the control group at M6. (B) The comparison of HADS-depression score change was decreased in the IPCP group compared with that in the control group. (C) No difference was observed in the percentage of depression evaluated by the HADS-depression score between the groups. (D) IPCP slightly decreased the depression grade at month 6 compared with the control group. ${ }^{*} \mathrm{P}<0.05$ vs. control. Comparison was performed using the $\mathrm{t}$-test or Chi-square test. Values are expressed as the mean \pm standard deviation. NS, no significance; IPCP, incremental patient care program; M, month; HADS, Hospital Anxiety and Depression Scale.

There was a significant negative correlation between HADS-A and QLQ-C30 function scores at baseline in the IPCP group $(\mathrm{P}=0.024)$, but not at 6 months.

\section{Discussion}

The major results of the present study were as follows: i) The IPCP group experienced an insignificant decrease in anxiety, as assessed by the HADS-A scores, and significantly reduced depression, as assessed by the HADS-D scores; ii) participants receiving IPCP experienced improved QoL, as assessed by the QLQ-C30.

Recently, the survival of CRC patients has been reported to be increased, while affected patients are at high risk of experiencing psychosocial problems (such as depression and anxiety), which has a negative impact on their health-associated QoL $(23,24)$. A previous clinical study illustrates that CRC patients exhibited an obviously higher prevalence of depression (19.0\% vs. $12.8 \%)$ and anxiety (20.9\% vs. $11.8 \%)$ compared with cancer-free age- and sex-matched individuals (23). Another study revealed a negative association between the patients' emotional functioning score and HADS-A score, as well as HADS-D score, and the correlation between HADS-D and QoL dimensions was significantly higher compared with the correlation between QoL and HADS-A (24). These previous clinical studies indicate that psychosocial disorders (including anxiety and depression) are conditions that negatively influence the QoL of CRC patients.

Based on several previous studies, HADS-A score, HADS-D score and the EORTC QLQ-C30 scale are reliable measurement tools for evaluating anxiety, depression and QoL of cancer patients $(25,26)$.

In clinical practice, care interventions have been confirmed to be beneficial for maintaining psychological health in cancer patients. For instance, one study reported that the health education level of patients prior to treatment initiation was associated with decreased anxiety and depression, as assessed by HADS-A/D scores (6). Another study revealed that, compared with the baseline, the HADS-D score was decreased in patients with CRC after a nurse-assisted screening and referral for participation in a care program (27). Although certain previous studies have demonstrated the positive impact of care interventions on mental health among patients with CRC, only few have investigated the effects of an IPCP on anxiety and depression in patients with CRC receiving adjuvant chemotherapy. In the present study, a novel IPCP was designed in three steps as follows: i) After a literature search, the common care methods for carcinoma were reviewed. ii) The detailed program was designed and three nurses with nursing experience of $>10$ years at the department of digestion were invited to rate and make decisions regarding the intervention program; the final result was selected as the final program. iii) A preliminary study was performed to confirm the effectiveness of the IPCP in 10 patients with CRC receiving adjuvant chemotherapy, and its efficacy was indicated to be good in these patients. The difference in the contents between IPCP and traditional care were that i) IPCP was performed more intensively, while, for traditional care, the post-operative medicine management and usual advice on post-operative rehabilitation were provided according to patients' requirements (28); ii) IPCP consisted of patient health education, physical exercise, telephone 
A

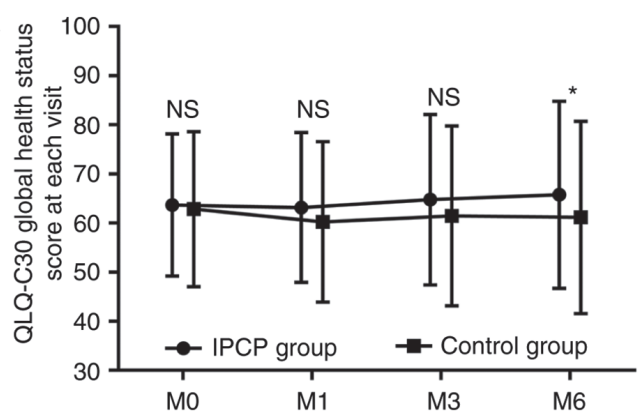

C

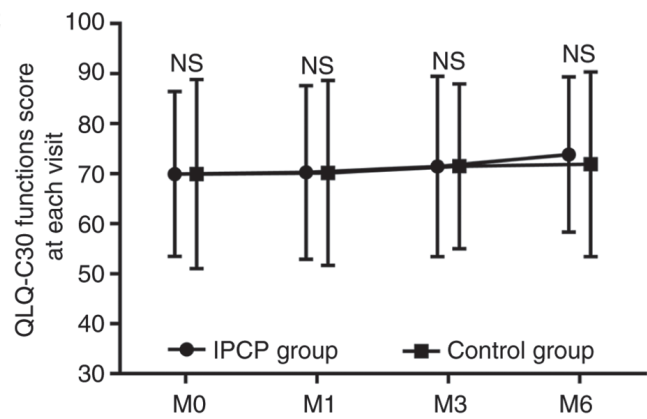

E

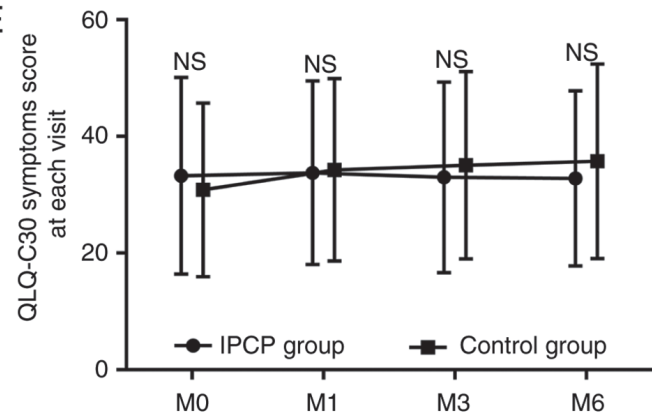

B

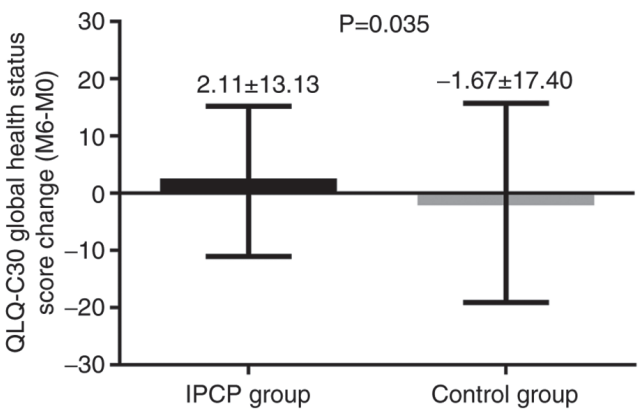

D

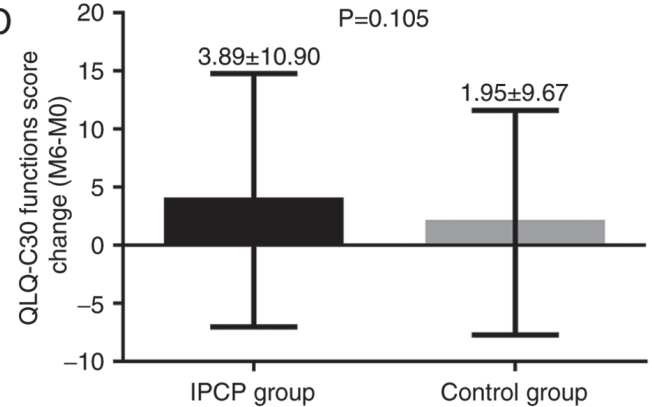

$\mathrm{F}$

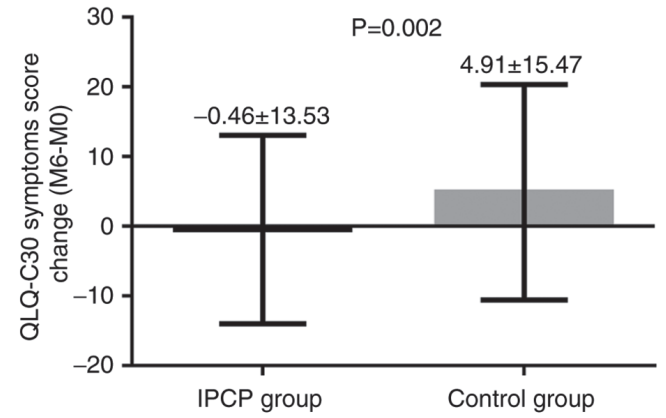

Figure 4. Comparison of quality of life between IPCP group and control group. (A) QLQ-C30 global health status score was higher in the IPCP group compared with that in the control group. (B) The QLQ-C30 global health status score change (M6-M0) was increased in the IPCP group compared with that in the control group. (C and D) No difference was observed between groups in QLQ-C30 function score (C) at each visit as well as (D) regarding its change. (E) No difference between groups was identified in the QLQ-C30 symptoms score at each visit. (F) The QLQ-C30 symptoms score change (M6-M0) was decreased in the IPCP group compared with that in the control group. Values are expressed as the mean \pm standard deviation. *P $<0.05$ vs. control. NS, no significance between the two groups at the same-time point; IPCP, incremental patient care program; M, month; QLQ-C30, European Organization for Research and Treatment of Cancer Quality of Life Questionnaire.

counseling, regular examination as well as care activities. iii) During the care activities, all caregivers were provided with lessons with regard to how to help patients complete their daily exercise. The present study demonstrated the possible efficacy of IPCP in slightly decreasing anxiety and reducing depression. The possible reasons were as follows: First, IPCP included regular patient health education, which may provide correct information and knowledge on risk factors, treatment and prognosis of CRC to patients, thereby decreasing their fear and confusion that may arise from inaccurate information and rumors from unreliable websites, thus reducing their risk of anxiety and depression. Furthermore, IPCP is a care intervention with low- and high-intensity physical exercise, and the physical exercise may promote the motor and sensory recovery thereby increasing patient confidence and reducing the risk of anxiety and depression (29). Finally, IPCP is a program for patients that relies on assistance from caregivers and nurses, thus providing more opportunities for patients to increase communication. Increased communication between patients and nurses may contribute to increased psychological health, including decreased anxiety and depression, among patients with CRC receiving adjuvant chemotherapy.

QoL is a critical outcome measure in the assessment of health status and treatment efficacy in different patients, in including patients with cancer and rheumatoid arthritis. According to accumulating evidence, favorable outcomes of care interventions regarding QoL have been identified in cancer patients. For instance, one previous study suggested that QoL, as assessed by the EORTC QLQ-C30 and EuroQol-5 dimensions scales, was improved among patients receiving chemotherapy for $\mathrm{CRC}$ after they received health education prior to treatment initiation (6). According to a review of 11 studies, various forms of psychosocial intervention, including health educational interventions, cognitive-behavioral therapy, relaxation exercise and supportive group therapy, contribute to a decreased length of hospital stay, decreased days to stoma proficiency and decreased hospital-associated anxiety and depression, as well as improved QoL outcomes in patients with CRC (30). Therefore, for patients 
TNM stage II patients
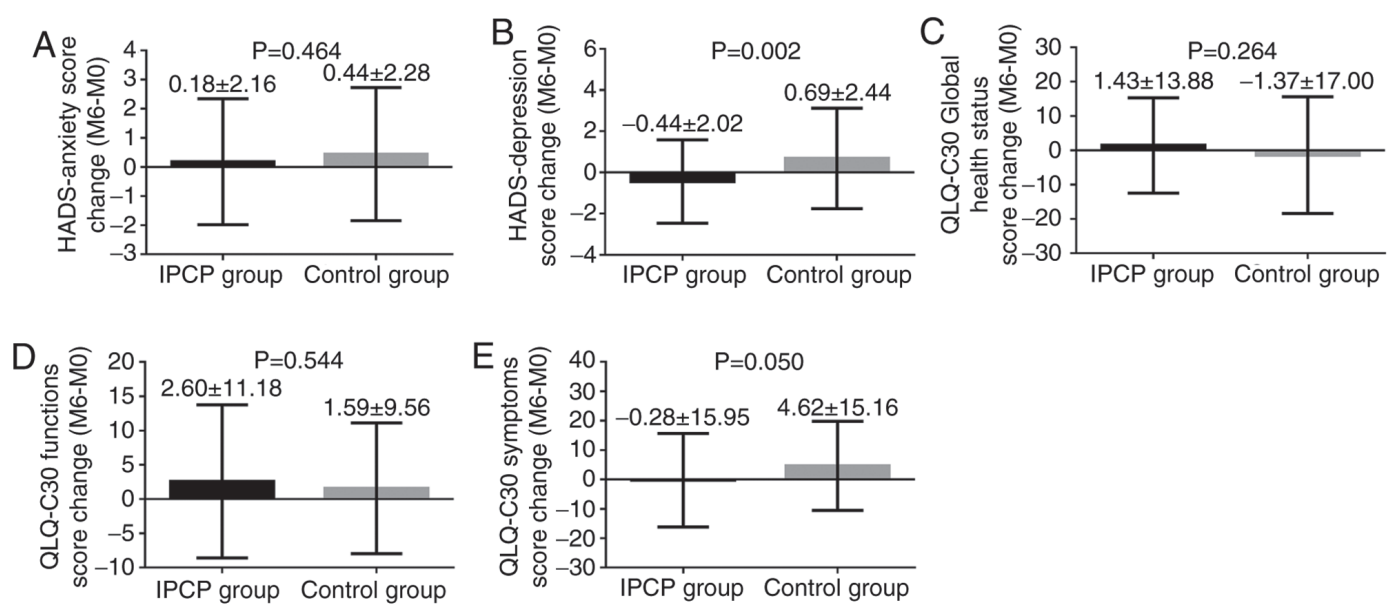

TNM stage III patients
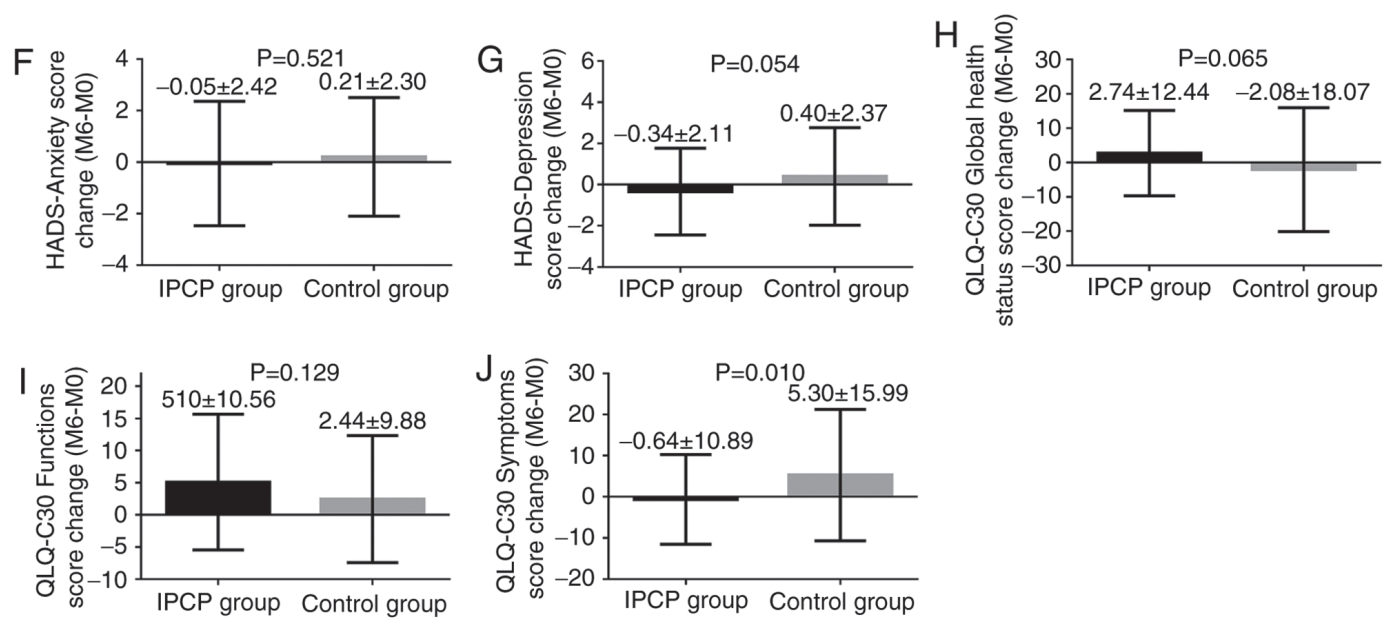

Figure 5. Subgroup analysis between TNM stage II and stage III patients. (A-E) For TNM stage II patients, no difference was observed in the change of (A) HADS-anxiety score, (C) QLQ-C30 global health status score and (D) QLQ-C30 function score from baseline (M0) to M6 between groups. (B) The HADS-depression score change (M6-M0) and (E) the QLQ-C30 symptoms score (M6-M0) was decreased in the IPCP group compared with those in the control group. (F-J) As for TNM stage III patients, (J) the QLQ-C30 symptoms score (M6-M0) was decreased in the IPCP group compared with that in the control group and (G) the HADS-depression score change (M6-M0) was numerically decreased, while (H) the QLQ-C30 global health status score change (M6-M0) was numerically increased in the IPCP group compared with that in the control group. (F and I) There was no difference in the change of HADS-anxiety score and QLQ-C30 functions score from baseline (M0) to M6 between groups. Values are expressed as the mean \pm standard deviation. NS, no significance; IPCP, incremental patient care program; M, month; HADS, Hospital Anxiety and Depression Scale; TNM, tumor-nodes-metastasis; QLQ-C30, European Organization for Research and Treatment of Cancer Quality of Life Questionnaire.

with CRC, care interventions have a positive effect regarding the improvement of QoL. In the present study, the influence of IPCP on QoL was also explored, and assessment via the QLQ-C30 global health status scores, QLQ-C30 function scores and QLQ-C30 symptom scores suggested that IPCP contributed to an improvement in QoL among patients with $\mathrm{CRC}$ receiving adjuvant chemotherapy. The first possible reason for this effect is that IPCP is a safe and effective way to provide low- and high-intensity physical exercise that contributes to functional recovery and to achieve long-term improvements in activities of daily living, thereby increasing physical health and improving QoL of patients with CRC. Furthermore, IPCP has an important role in maintaining a good environment for increasing communication among patients, family members and nurses, thereby promoting mutual understanding and cooperation and decreasing anxiety and depression, thus improving mental health and increasing QoL.
Of note, the present study had certain limitations. First, the sample size was relatively small, leading to low statistical power. Furthermore, all patients enrolled in the present study were from a single centre; thus, further studies should be performed recruiting patients from multiple centres. Finally, the duration of follow-up was relatively short; thus, the long-term effects on IPCP on anxiety, depression and QoL in patients with CRC remain elusive.

In conclusion, IPCP led to a slight decrease in anxiety to a certain extent and contributed to a significant reduction in depression and an improvement in QoL in patients with CRC receiving adjuvant chemotherapy. The present study provided a novel IPCP that may serve as an efficient care program for outcome improvements in CRC.

\section{Acknowledgements}

Not applicable. 


\section{Funding}

No funding was received.

\section{Availability of data and materials}

The datasets used and/or analyzed during the current study are available from the corresponding author on reasonable request.

\section{Authors' contributions}

XL designed the experiment, JL and XL performed the experiments and wrote the manuscript, and XL analyzed the data and revised the manuscript.

\section{Ethics approval and consent to participate}

The present study was performed according to the tenets established in the Declaration of Helsinki and was approved by the Ethics Committee of the 2nd Affiliated Hospital of Harbin Medical University (Harbin, China). Written informed consent was obtained from all patients.

\section{Patient consent for publication}

Not applicable.

\section{Competing interest}

The authors declare that they have no competing interests.

\section{References}

1. Brenner H, Kloor M and Pox CP: Colorectal cancer. Lancet 383 : 1490-1502, 2014.

2. Ferlay J, Shin HR, Bray F, Forman D, Mathers C and Parkin DM: Estimates of worldwide burden of cancer in 2008: GLOBOCAN 2008. Int J Cancer 127: 2893-2917, 2010.

3. Torre LA, Bray F, Siegel RL, Ferlay J, Lortet-Tieulent J and Jemal A: Global cancer statistics, 2012. CA Cancer J Clin 65: 87-108, 2015.

4. Siegel R, Ma J, Zou Z and Jemal A: Cancer statistics, 2014. CA Cancer J Clin 64: 9-29, 2014.

5. Gonzalez-Saenz de Tejada M, Bilbao A, Baré M, Briones E, Sarasqueta C, Quintana JM and Escobar A; CARESS-CCR Group: Association between social support, functional status, and change in health-related quality of life and changes in anxiety and depression in colorectal cancer patients. Psychooncology 26: 1263-1269, 2017.

6. Polat U, Arpaci A, Demir S, Erdal S and Yalcin S: Evaluation of quality of life and anxiety and depression levels in patients receiving chemotherapy for colorectal cancer: Impact of patient health education before treatment initiation. J Gastrointest Oncol 5: 270-275, 2014

7. Wan Puteh SE, Saad NM, Aljunid SM, Abdul Manaf MR, Sulong S, Sagap I, Ismail F and Muhammad Annuar MA Quality of life in Malaysian colorectal cancer patients. Asia Pac Psychiatry 5 (Suppl 1): S110-S117, 2013.

8. Tsunoda A, Nakao K, Hiratsuka K, Yasuda N, Shibusawa M and Kusano M: Anxiety, depression and quality of life in colorectal cancer patients. Int J Clin Oncol 10: 411-417, 2005.

9. Alacacioglu A, Binicier O, Gungor O, Oztop I, Dirioz M and Yilmaz U: Quality of life, anxiety, and depression in Turkish colorectal cancer patients. Support Care Cancer 18: 417-421, 2010.

10. Medeiros M, Oshima CT and Forones NM: Depression and anxiety in colorectal cancer patients. J Gastrointest Cancer 41: 179-184, 2010.
11. Demark-Wahnefried W and Jones LW: Promoting a healthy lifestyle among cancer survivors. Hematol Oncol Clin North Am 22: 319-342, 2008.

12. Morey MC, Snyder DC, Sloane R, Cohen HJ, Peterson B, Hartman TJ, Miller P, Mitchell DC and Demark-Wahnefried W: Effects of home-based diet and exercise on functional outcomes among older, overweight long-term cancer survivors: RENEW: A randomized controlled trial. JAMA 301: 1883-1891, 2009.

13. Allgayer H, Owen RW, Nair J, Spiegelhalder B, Streit J, Reichel C and Bartsch H: Short-term moderate exercise programs reduce oxidative DNA damage as determined by high-performance liquid chromatography-electrospray ionization-mass spectrometry in patients with colorectal carcinoma following primary treatment. Scand J Gastroenterol 43: 971-978, 2008.

14. XiaoF, Song X,Chen Q, Dai Y,Xu R, Qiu Cand Guo Q: Effectiveness of psychological interventions on depression in patients after breast cancer surgery: A meta-analysis of randomized controlled trials. Clin Breast Cancer 17: 171-179, 2017.

15. Li Z, Geng W, Yin J and Zhang J: Effect of one comprehensive education course to lower anxiety and depression among Chinese breast cancer patients during the postoperative radiotherapy period-one randomized clinical trial. Radiat Oncol 13: 111, 2018.

16. Edge SB, Byrd DR, Compton CC, Fritz AG, Greene FL and Trotti A, III: AJCC Cancer Staeging Manual. 7th ed. New York: Springer, 2010.

17. Seligman MEP, WalkerEF and Rosenhan DL. Abnormal Psychology (4th Edition). W.W. Norton \& Company, New York, NY, 2001.

18. Diagnostic and Statistical Manual of Mental Disorders, Fifth Edition (DSM-5). American Psychiatric Association 2013.

19. Zigmond AS and Snaith RP: The hospital anxiety and depression scale. Acta Psychiatr Scand 67: 361-370, 1983.

20. Barcaccia B: 'Quality Of Life: Everyone Wants It, But What Is It?'. 2016.

21. Tung HY, Chao TB, Lin YH, Wu SF, Lee HY, Ching CY, Hung KW and Lin TJ: Depression, fatigue, and QoL in colorectal cancer patients during and after treatment. West J Nurs Res 38: 893-908, 2016.

22. Van Vulpen JK, Velthuis MJ, Steins Bisschop CN, Travier N, Van Den Buijs BJ, Backx FJ, Los M, Erdkamp FL, Bloemendal HJ, Koopman M, et al: Effects of an exercise program in colon cancer patients undergoing chemotherapy. Med Sci Sports Exerc 48: 767-775, 2016.

23. Mols F, Schoormans D, de Hingh I, Oerlemans S and Husson O: Symptoms of anxiety and depression among colorectal cancer survivors from the population-based, longitudinal PROFILES Registry: Prevalence, predictors, and impact on quality of life. Cancer 124: 2621-2628, 2018.

24. Aminisani N, Nikbakht $H$, Asghari Jafarabadi $M$ and Shamshirgaran SM: Depression, anxiety, and health related quality of life among colorectal cancer survivors. J Gastrointest Oncol 8: 81-88, 2017.

25. Thomas BC, Devi N, Sarita GP, Rita K, Ramdas K, Hussain BM, Rejnish R and Pandey M: Reliability and validity of the Malayalam hospital anxiety and depression scale (HADS) in cancer patients. Indian J Med Res 122: 395-399, 2005.

26. Matsumoto T, Ohashi Y, Morita S, Kobayashi K, Shibuya M, Yamaji Y, Eguchi K, Fukuoka M, Nagao K, Nishiwaki Y, et al: The quality of life questionnaire for cancer patients treated with anticancer drugs (QOL-ACD): Validity and reliability in Japanese patients with advanced non-small-cell lung cancer. Qual Life Res 11: 483-493, 2002.

27. Livingston PM, Craike MJ, White VM, Hordern AJ, Jefford M, Botti MA, Lethborg C and Oldroyd JC: A nurse-assisted screening and referral program for depression among survivors of colorectal cancer: Feasibility study. Med J Aust 193 (5 Suppl): S83-S87, 2010.

28. Watanabe T, Itabashi M, Shimada Y, Tanaka S, Ito Y, Ajioka Y, Hamaguchi T, Hyodo I, Igarashi M, Ishida H, et al: Japanese society for cancer of the colon and rectum (JSCCR) guidelines 2014 for treatment of colorectal cancer. Int J Clin Oncol 20: 207-239, 2015.

29. Sheiner LB: Is intent-to-treat analysis always (ever) enough? Br J Clin Pharmacol 54: 203-211, 2002.

30. Hoon LS, Chi Sally CW and Hong-Gu H: Effect of psychosocial interventions on outcomes of patients with colorectal cancer: A review of the literature. Eur J Oncol Nurs 17: 883-891, 2013.

This work is licensed under a Creative Commons Attribution-NonCommercial-NoDerivatives 4.0 International (CC BY-NC-ND 4.0) License. 